\title{
Is 'happy hypoxia' in COVID-19 a disorder of autonomic interoception? A hypothesis
}

\author{
Alejandra González-Duarte ${ }^{1}$ - Lucy Norcliffe-Kaufmann ${ }^{2}$
}

Received: 23 June 2020 / Accepted: 8 July 2020 / Published online: 15 July 2020

(c) Springer-Verlag GmbH Germany, part of Springer Nature 2020

Keywords COVID-19 · Hypoxia · Dyspnea · "Happy hypoxemia"

Dear Editors,

One of the aspects of coronavirus disease 2019 (COVID-19) puzzling clinicians coping with management of the pneumonia that one of the disease's complications is the presentation of patients with extremely low blood oxygenation, but no sensation of dyspnea [1]. This phenomenon has given rise to the term "happy hypoxemia" [1]. In the Wuhan cohort of patients infected with severe acute respiratory syndrome coronavirus 2 (SARS-COV-2), only 19\% complained of shortness of breath; $62 \%$ of those with severe disease and $46 \%$ of those who ended up intubated, ventilated or dead did not present with dyspnea [2]. What strikes us as odd, is that these patients are tachycardic with tachypnea and respiratory alkalosis. These signs suggest that at least some sensory information must reach the brainstem to elicit a partial compensatory reflex respiratory response that is sufficent to lower the $\mathrm{CO}_{2}$ level, which diffuses more rapidly across the alveoli than oxygen. However, these patients have no conscious awareness of hypoxia.

The homeostatic afferent information emanating from the body forms part of our interoceptive system, which senses the body's physiological condition, creates awareness, and leads to conscious feelings or symptoms [3]. This process occurs via projections from the brainstem to the cortex that allow the brain to process homeostatic afferent signals. When the brain receives the signal of internal hypoxia, it

Alejandra González-Duarte

gonzalezduarte@aol.com

1 Department of Neurology, Instituto Nacional de Ciencias Médicas y Nutrición Salvador Zubirán, Vasco de Quiroga 15, Col. Belisario Dominguez Sección XVI, Tlalpan, 14080 Mexico City, Mexico

2 Dysautonomia Center, New York University School of Medicine, New York, NY, USA gives rise to the sensation of "air hunger" and a need to breathe, which is curiously absent in severe COVID-19 patients.

The respiratory responses to hypoxia occur due to the presence of sensory nerves in chemoreceptive areas. These recognize the shift in the internal environment, relay the information to the brainstem, and stimulate an increase in the ventilatory drive. Respiratory pathology elicits autonomic reflexes, such as bronchospasm, secretions, or cough. Dyspnea is the conscious distressing symptom of difficulty in breathing that can be triggered by many clinical conditions [4]. In the setting of cardiopulmonary illness, dyspnea arises from inputs from multiple homeostatic afferents. Interoceptive processing of these signals create a sense of shortness of breath and the urge to breathe. This primitive brainstem reflex is essential for survival as it can respond to a wide range of stimuli, including hypoxia, hypercapnia, irritants, acidosis, airway collapse, and pulmonary vascular congestion.

The glossopharyngeal afferents innervating the carotid body, and the vagal afferents innervating the respiratory tract, play a vital role in monitoring organ function and controlling body homeostasis through activation of the autonomic nervous system. These neurons are the primary sensory inputs of a series of reflex circuits that control key visceral functions, including blood pressure, swallowing, gastrointestinal motility, airway caliber, and tidal volume [4]. They also produce the first afferents for the conscious sensation of dyspnea.

Mechanical or chemical stimuli of pulmonary receptors expressed on afferent vagal nerve terminals in the lung arrive in the brainstem through small-diameter myelinated (Aס)- or unmyelinated (C)-fiber nerve axons with cell bodies in the jugular or nodose ganglia of the vagus (Fig. 1). Both jugular and nodose pulmonary C-fiber afferents respond to inflammatory mediators and tissue acidification 


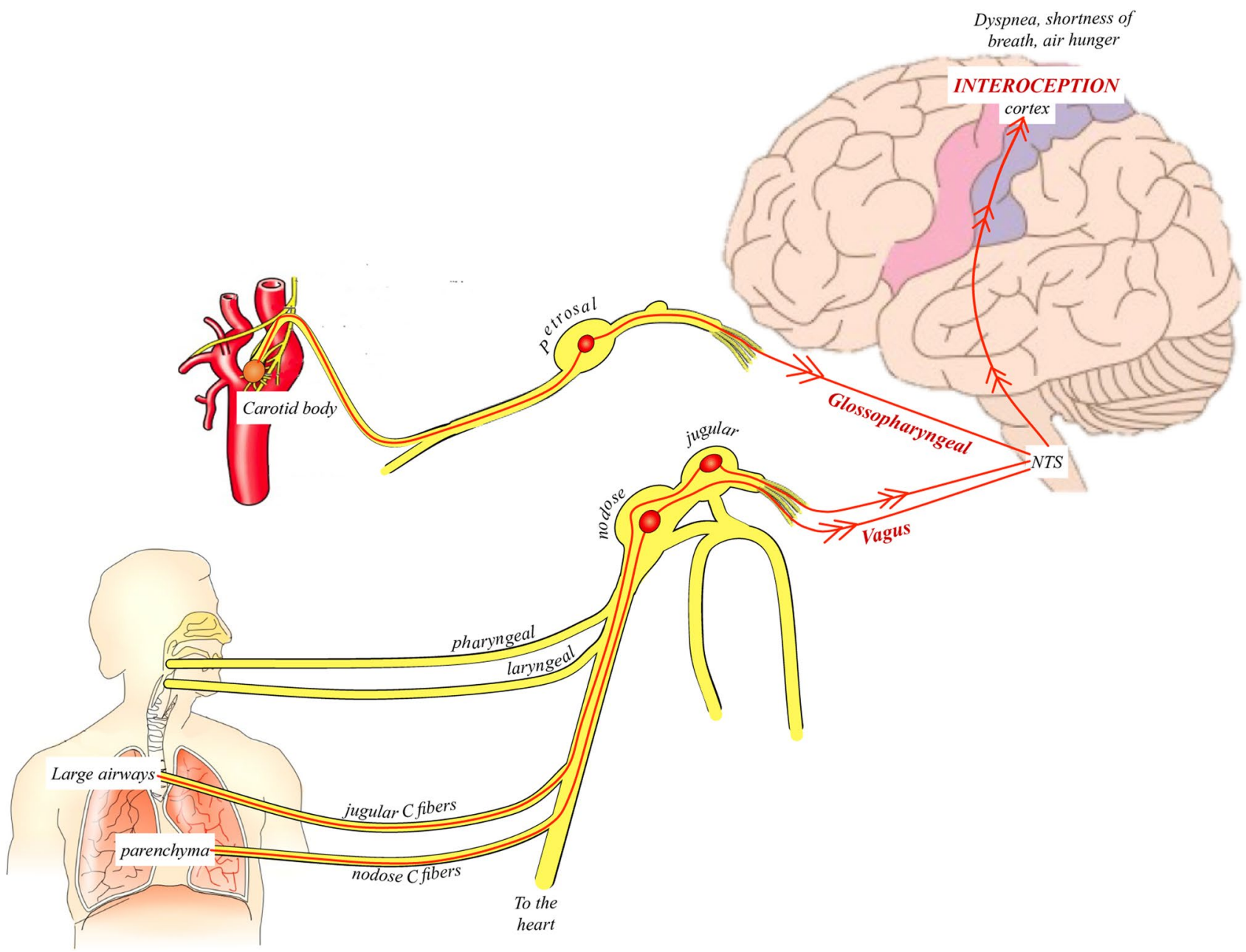

Fig. 1 Neurophysiology of dyspnea. Main afferent (sensory) homeostatic information arising from areas of the vasculature and lungs give rise to the sensation of dyspnea. When stimulated, the chemoreceptive and mechanoreceptive signals are transmitted to the brainstem via the glossopharyngeal and vagus nerves, converging at the nucleus of the tractus solitarus (NTS). Subsequent projections continue to the

in a graded fashion; these can be considered "nociceptive" fibers as they do not react to eupneic breathing or other regular events, but are excited by "noxious" or "potentially noxious" stimuli. The jugular and nodose nerve fibers of the lung have distinct differences in terms of their embryologic origin, pharmacological responses, and neurochemistry. Thus, they serve different functions-which are hard to tease apart in the intact human. The nodose C-fibers probably play a more prominent role in the genesis of dyspnea and the subjective sensation of breathing difficulty. In contrast, jugular fibers may play a more prominent role in coughing [4]. The vagal C-fiber afferents innervate the larynx response within seconds to laryngeal discomfort and appear to be important in stimulating cough. Meanwhile, the dyspneic sensation is specifically somatosensory cortex and other higher brain regions, which provide the interoceptive sense of the internal environment of the body. The processing of these signals within the cortex gives rise to sensations such as air hunger, dyspnea, or shortness of breath. This interceptive processing appears to be abnormally blunted in patients with coronavirus disease 2019

related to the activation of a subgroup of nodose vagal afferent that express adenosine receptors. The afferent information arriving from the vagal and glossopharyngeal nerves converges at the nucleus of the tractus solitarius in the medulla, a key relay site for a variety of other critical homeostatic signals. From here, there are connections to the higher centers of the brain towards the thalamus, somatosensory cortex, insular cortex, and amygdala, all involved in the perception of breathing.

The pathophysiology underlying the dissociation between profound hypoxemia and overt dyspnea in COVID-19 pneumonia is, at this point, unclear. In our experience, this disassociation exists in patients with severe lesions in the glossopharyngeal or vagus nerves due to damage to the cranial nerve after neck cancer or congenital neuropathies, but these 
findings are unexpectedly absent in the autopsy reports that are now emerging in COVID-19 cases.

The possibility that the novel SARS-COV-2 is neuroinvasive remains controversial. On one hand, in severe COVID-19 cases, neurological symptoms, such as anosmia, headache, altered mental status, seizures, and delirium, are common; and SARS-COV-2 is found in the cerebral spinal fluid and thought to enter the brain through synapseconnected routes [1]. The possible damage to the afferent hypoxia-sensing neurons in persons with COVID-19 could be due to the intense cytokine storm or the direct effect of SARS-COV2 on mitochondria or on the nerve fibers [1]. On the other hand, the findings from brain magnetic resonance imaging (MRI) studies and pathology reports in lethal COVID-19 cases are inconsistent and do not provide a pathophysiological correlate to explain the absence of dyspnea [5]. The common brain pathology findings in fatal COVID-19 cases are multiple areas of ischemic and microbleeding hemorrhagic strokes with only small regions of inflammation; however, it is worth noting that at least $40 \%$ of cases brain imaging studies were normal and there were no signals of brainstem abnormalities on the MRI scans. The neurological manifestations of other coronaviruses are even less well studied, but neuropathy and myopathy are reported in a handful of cases of both severe acute respiratory syndrome (SARS-CoV) and middle eastern respiratory syndrome (MERS-CoV). What makes COVID-19 most intriguing at this point is what the patient does not sense and what the brain does not show in terms of pathology.

Regardless of the uncertain underlying pathology, reduced perception of dyspnea is a disorder of blood-gas interoception. It may mask the severity of the medical status and ultimately delay patients from seeking urgent medical care. Patients admitted with COVID-19 can suffer sudden death after voluntary "breaks" from the oxygen supplementation. Recognizing "happy hypoxia" as a feature of COVID-19 pneumonia has led to better patient care, with physicians relying on other markers of disease, such as tachycardia, fever, or serum inflammatory acute reactants, to guide treatment or discharge patients from the hospital. Continuing research on how the novel coronavirus impacts peripheral sensors and neural pathways holds the promise of further clarifying its mechanisms.

Acknowledgements We are thankful to Horacio Kaufmann for his helpful discussion of the manuscript.

Author contributions All authors contributed equally to this article.

Funding The authors received no funding for this article.

\section{Compliance with ethical standards}

Conflict of interest The authors of this article have nothing to disclose.

\section{References}

1. Archer SL, Sharp WW, Weir EK (2020) Differentiating COVID19 pneumonia from acute respiratory distress syndrome (ARDS) and high altitude pulmonary edema (HAPE): therapeutic implications. Circulation. https://doi.org/10.1161/CIRCULATIO NAHA.120.047915

2. Wei-jie G, Zheng-yi N, Yu H et al (2020) Clinical characteristics of Coronavirus disease 2019 in China. N Engl J Med 382:1708-1720

3. Robinson D, Gebhart F (2008) Inside information-the unique features of visceral sensation. Mol Interv 8(5):242-253

4. Burki N, Lee L (2010) Mechanisms of dyspnea. Chest 1385:1196-1201

5. Coolen T, Lolli V, Sadeghi N, et al. (2020) Early postmortem brain MRI findings in COVID-19 non-survivors. Neurology. https://doi. org/10.1212/WNL.0000000000010116 